\title{
Super-resolution method for arbitrary retrospective sampling in fluorescence tomography with raster scanning photodetectors
}

\author{
Xiaofeng Zhang* \\ Department of Radiology, Duke University Medical Center, Durham, NC, USA 27710 \\ *steve.zhang@duke.edu; phone 1919 257-8564; fax 1919 684-2711
}

\begin{abstract}
Dense spatial sampling is required in high-resolution optical imaging and many other biomedical optical imaging methods, such as diffuse optical imaging. Arrayed photodetectors, in particular charge coupled device cameras are commonly used mainly because of their high pixel count. Nonetheless, discrete-element photodetectors, such as photomultiplier tubes, are often desirable in many performance-demanding imaging applications. However, utilization of the discrete-element photodetectors typically requires raster scan to achieve arbitrary retrospective sampling with high density. Care must be taken in using the relatively large sensitive areas of discrete-element photodetectors to densely sample the image plane. In addition, off-line data analysis and image reconstruction often require full-field sampling. Pixel-by-pixel scanning is not only slow but also unnecessary in diffusion-limited imaging. We propose a superresolution method that can recover the finer features of an image sampled with a coarse-scale sensor. This generalpurpose method was established on the spatial transfer function of the photodetector-lens system, and achieved superresolution by inversion of this linear transfer function. Regularized optimization algorithms were used to achieve optimized deconvolution. Compared to the uncorrected blurred image, the proposed super-resolution method significantly improved image quality in terms of resolution and quantitation. Using this reconstruction method, the acquisition speed with a scanning photodetector can be dramatically improved without significantly sacrificing sampling density or flexibility.
\end{abstract}

Keywords: Fluorescence tomography, diffuse optical imaging, data sampling, super-resolution, deconvolution, motion deblurring, reconstruction

\section{INTRODUCTION}

A central question in biomedical imaging technology development is the sensitivity of the method, which is typically established on the characteristics of the particular detection technology. This is especially true in biomedical optical imaging, in which direct detection is often the approach for signal acquisition. As a result, the performance of imaging instrument and methodology were directly associated to the specific hardware used (or, assumed) in the studies, e.g., the results demonstrated in [1-5]. Under many circumstances, one wishes to incorporate desired features of advanced detection technologies in new applications to create novel capabilities. However, these attempts are often hindered by practical limitations of the established methodologies and protocols.

In this study, our goal is to investigate the feasibility of utilizing advanced discrete-element photodetectors in imaging applications where arrayed photodetectors are conventionally required. To accomplish this goal, we have developed new techniques for signal acquisition, data processing, and image reconstruction. This new method was developed with the intention to advance the emerging biomedical imaging technology of diffuse optical imaging. The family of diffuse optical imaging technology includes diffuse optical tomography, fluorescence molecular tomography (aka, fluorescence diffuse optical tomography), and bioluminescence tomography, all of which share the same physical principles of diffusive photon migration in highly scattering biological tissue [6-9].

Currently, diffuse optical imaging methods typically use lens-coupled arrayed or fiber-coupled discrete-element photodetectors. In recent years, dramatically increased attentions have been drawn to arrayed photodetectors, e.g., charge coupled device (CCD), intensified CCD (ICCD), electron multiplying CCD (EMCCD), and scientific complementary metal-oxide-semiconductor (sCMOS). These devices have remarkably high sampling density and are easy to operate in biomedical imaging experiments, particularly for noncontact and multimodality imaging [10-14]. However, compared to the discrete-element photodetectors, such as photomultiplier tubes (PMTs) and avalanche photodiodes (APDs), the 
arrayed photodetectors sometimes can only offer limited performance in key parameters, e.g., sensitivity, dynamic range, temporal resolution, and noise level.

These parameters are especially critical when it applies to in vivo imaging. In these applications, the effective signal intensity (i.e., the number of useful photons per unit time) is typically very low, which requires long integration time. This limitation is further amplified in time- or spectro-resolved acquisition methods, e.g., time-gated [14-19] and hyperspectral [20-23] imaging. However, in vivo biomedical applications require fast acquisition time to capture dynamic biological processes and to minimize the artifacts due to physiological motion (e.g., respiration and heart beat). These conflicting requirements place high demands on the detection performance (particularly sensitivity), which are often not achievable using arrayed photodetectors with current technology.

Our method is to use a discrete-element photodetector with desired photoelectrical characteristics to produce full-field image by mechanical scan. The optical signal is coupled to the photodetector via a widefield imaging lens, in order to maintain the flexibility offered by camera-based configurations. By scanning the photodetector in the image plane of the lens, full-field image of the object is obtained. From the perspective of the instrument user, the new imaging instrument operates in a similar manner as the conventional camera-based instruments but provides desired new imaging features, thereby enabling novel imaging applications.

The proposed method introduces two main sources of imaging artifacts: (1) motion artifact as a result of the scanning photodetector and (2) spatial modulation because of the optical characteristics of the specific photodetector and imaging lens. Although motion artifact can be effectively eliminated by stopping the scan head during data acquisition, the negative consequence is increased acquisition time, a significant overhead in high-density sampling. The other source of imaging artifact stems from the spatial transfer function of the specific photodetector-lens system. Because the geometry and sensitivity profile of the photodetector's active area is typically prescribed by the detection technology and the device manufacturer, this transfer function varies widely and has very limited degree of freedom. As a result of both sources of artifact, image resolution and, more importantly, accuracy are compromised. Similar problems exist in other disciplines and have been studied extensively, e.g., in microscopy [24], medical imaging [25], and remote sensing [26]. However, its new application in diffuse optical imaging has its unique characteristics that are worth special considerations, as presented in this work.

\section{METHODOLOGY}

\subsection{Sampling in the image plane}

The photodetector samples in the image plane of a widefield imaging lens. Compared to the conventional methods that sample on the object surface directly or via fixed coupling lenses, the proposed method has the following advantages: (1) noncontact acquisition greatly simplifies experimental setup for in vivo applications; (2) sampling density can be controlled over a broad range by adjusting the scanning density and lens focus; and (3) availability of large collections of standard imaging lenses (e.g., for microscopy and photography) allows highly flexible experimental configurations without resorting to time-consuming and costly customized optics.

However, it is well known that the main limitation of this type of sampling method is speed. In order to expedite acquisition, continuous scanning is used. Compared to the pixel-by-pixel point-scan technique, the line-scan technique has the advantage of fast scanning speed because the overhead for actuators is eliminated. The actuator overhead includes electrical communications between the computer and the controller, and mechanical acceleration/deceleration of the scan head. For typical stepper/servo motors, the actuator overhead is on the order of milliseconds. For a $100 \times 100$ point scan, for example, the accumulated actuator overhead is on the order of tens of seconds, a significant burden for in vivo experiments.

In a standard raster scan paradigm, the scan head returns to its origin along the axis of scanning after each line-scan, aka, homing. As a result, the photodetector is either idle or acquiring unusable data during approximately half of the scan time. We adopted a bi-directional raster scan pattern that nearly doubles the scan efficiency by eliminating device homing. The schematics of actuator motion and photodetector timing are shown in Figure 1.

Knowing the exact timing of the scanner and the acquisition hardware, one can retrospectively map the sampling locations to time in the time-resolved data. Without losing generality, one can cast this one-dimensional data into an image with $x \times y$ pixels, where $x$ is the number of pixels along the line-scan direction and $y$ is the number of scan lines. Specifically, the complete time-resolved one-dimensional data is first segmented by the number of scan lines ( $y$-steps); 
and then, each data segment is divided into $x$ time-bins. The averaged values of these time-bins are assigned to the corresponding pixels in the resulting image. Note that the data lines corresponding to even-numbered line scans are flipped to account for the bi-directional scan pattern.
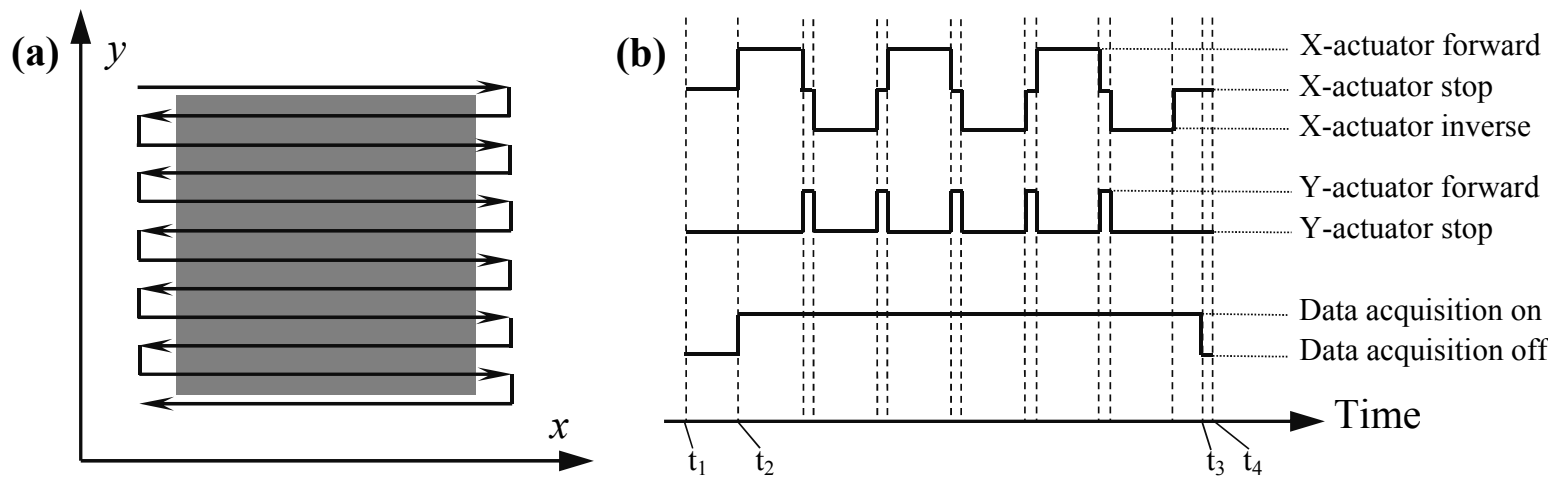

Figure 1. Schematics of the scan pattern and device timing. (a) Bi-directional raster scan, where the gray area represents the region of interest within the image plane, and the folded arrow-line represents the trajectory of the scan head. (b) Schematics of device timing of the linear actuators and the photodetector: $t_{1}$ - experiment starts, $t_{2}$ - data acquisition and raster scan start, $\mathrm{t}_{3}$ - data acquisition ends, and $\mathrm{t}_{4}$ - experiment ends. During data acquisition, the $x$-actuator moves continuously and the $y$-actuator moves at discrete steps.

A unique feature of the proposed continuous raster scan method is that the number of pixels in the $x$-dimension can be specified arbitrarily as long as the pixel-wise signal intensity is sufficiently higher than the sampling noise. This feature enables retrospective determination of image resolution and therefore allows more flexibility in experimental designs. In addition, because the $x$-resolution can be determined retrospectively (but subject to the limit of noise), the acquisition time is linearly related to image resolution (i.e., the number of scan lines).

Note that timing jitter of the hardware clocks and the actuators should be considered in practical experiments. These parameters can be quantified by experiments following established calibration principles for scanning devices. In this simulation study, however, we assume that the exact timing of scan and acquisition is known and well synchronized.

\subsection{System transfer function}

In conventional methods, the photodetector is stationary relative to the object, or the relative motion is small compared to the resolution (pixel size) of the photodetector. In the proposed method, however, the photodetector moves continuously during data acquisition. In principle, the velocity of motion is dependent on the intensity of optical signal. For brightfield imaging, the velocity is potentially only limited by the speed of the scanner (e.g., the linear actuators). As a result, motion artifacts are non-trivial and affect the image quality significantly.

Because the photodetector moves at discrete steps in the $y$-dimension (line-scan in the $x$-dimension), image blurring in the $y$-dimension is caused mainly by overlapping of photodetector coverage. In this study, we assume the scan head moves in the $y$-dimension at a step smaller than the $y$-dimension of the photodetector's active area (i.e., overlapped line scans). This is a condition should be maintained in practical imaging experiments because otherwise signals between scan lines will be unrecoverable.

In the $x$-dimension, however, the photodetector travels continuously at a known velocity. The transfer function is therefore the spatial convolution of the photodetector with the true image in the $x$-dimension, and modulated by the velocity of the scan head. Specifically, the modulated signal intensity is given by the convolution of the photodetector sensitivity function and the distance it travels

$$
s=f \otimes d
$$

where $s$ is the detected signal intensity, $f$ is the spatial point-spread function of the photodetector, and $d$ is the travel distance of the scan head during the time interval (weighted by the scan velocity). 
In our numerical simulations, the forward problem was formulated slightly differently from Equation (1) because the data was represented and processed in digital format. The continuously acquired time-resolved analog signal is related to the digital signal by weighted time-averaging

$$
s(x)_{t_{1}, t_{2}}=\left[\int_{t_{1}}^{t_{2}} s(x, t) v(t) d t\right] /\left(t_{2}-t_{1}\right)
$$

where $s(x)_{t_{1}, t_{2}}$ is the detected spatial-dependent signal time-averaged over the scan interval of $\left(t_{1}, t_{2}\right), s(x, t)$ is the spatial-/time-dependent analog signal, $v(t)$ is the scan velocity, and $t_{1}<t<t_{2}$ is scan time.

If one assumes that the photodetector has a detection width of $w$ pixels and a scanning distance of 1 pixel during the time interval of $\left(t_{1}, t_{2}\right)$, the spatial transfer function of the photodetector has a width of $\bar{w}=2 \times \operatorname{ceil}(w / 2)+1$, where $\operatorname{ceil}(k)$ is the ceiling-rounded value of $k$. Note that the time interval can always be adjusted to maintain an unity value for the scanning distance. In this study, we assume that the sensitivity function of the photodetector is boxcar-shaped, and that the scanning velocity is constant.

The pixel-wise digital signal intensity in the $x$-dimension is eventually given by

$$
s(i)=f(x) \otimes s(x)_{t_{i 1}, t_{i 2}}
$$

where $i$ is the index of pixel, $f(x)$ is the spatial transfer function of the photodetector, and $\left(t_{i 1}, t_{i 2}\right)$ is the time interval that the photodetector remains within the boundaries of the $i$-th pixel.

In the $y$-dimension, the signal intensity is given by the algebraic product of the photodetector sensitivity function and the true image. In the case of boxcar-shaped sensitivity function, the pixel-wise signal intensity is simply the onedimensional sum of its neighboring pixels in the true image.

Overall, image blurring can be formulated as the product of the system transfer function and the true image. This transfer function can be determined based on the knowledge of the photodetector sensitivity function and scan parameters, all of which are measurable or known.

\subsection{Super-resolution reconstruction}

The super-resolution reconstruction problem can be formulated as an inversion of the forward image blurring problem. The overall formulation of the image blurring problem described in Section 2.2 can be written in a vector format

$$
\hat{S}=F S
$$

where $\hat{S}$ is the blurred image, $F$ is the system transfer function, and $S$ is the true image.

The system transfer function is a $m \times n$ matrix, where $m$ is the number of total scan steps, and $n$ is the number of pixels in the image. $\hat{S}$ is a $m \times 1$ vector. $S$ is a $n \times 1$ vector. Note that if $m<n$, the inverse problem is underdetermined; and if $m>n$, it becomes over-determined. In a deblurring problem, one would like to reconstruct the true image $S$ from the blurred image $\hat{S}$ with knowledge of the system transfer function $F$. If $m=n$, a variety of direct inversion methods can be used to precisely recover the true image $S$.

In actual experiments, the number of scan steps is rarely equal to the number of pixels; and because the sensitive area of the photodetector is much smaller than that of the image, the system transfer function is highly sparse. In addition, sampling noise is always present in the measured data, changing the formulation of the image blurring problem into

$$
\hat{S}=F S+E
$$

where $E$ is a $m \times 1$ vector representing measurement error.

As a result, regularized inversion methods must be used. In this type of regularized reconstruction methods, the inversion problem is converted to an optimization problem that seeks to minimize a cost function defined by a specific set of regularization criteria.

We applied our previously developed regularized iterative inversion algorithm based on a conjugate gradient method [4], which minimizes the following cost function

$$
H=\|F \tilde{S}-\hat{S}\|_{2}^{2}+a\|\tilde{S}\|_{2}^{2}+b\|\nabla \tilde{S}\|_{2}^{2}
$$


where $a$ and $b$ are the regularization parameters, $\hat{S}$ is the measured data, $\tilde{S}$ is the intermediate result, and $\|F\|_{2}^{2}$ is the L-2 norm of matrix $F$. Within the cost function $H$, the first term regulates image fidelity (i.e., data error $E$ ); the second term regulates total image data size; and the third term regulates the smoothness of the image. By adjusting these regularization parameters, the reconstruction quality can be optimized.

\section{RESULTS AND DISCUSSION}

We performed two sets of simulations to validate the proposed method: the first one demonstrated the validity of the super-resolution method on two-dimensional imaging phantoms; and the second one demonstrated the capability of this method on diffuse optical imaging applications using a three-dimensional phantom.

All of the image reconstruction algorithms and data processing routines were implemented on the scientific computing software platform MATLAB (MathWorks, Natick, MA). Because of the large size of the system transfer matrices, sparse representations were used in data processing for large matrices.

\subsection{Two-dimensional phantoms}

In the first set of numerical simulations, we used two synthesized images as the imaging phantoms, Figures 2 . We first adopted a binary imaging resolution target to demonstrate the qualitative super-resolution/de-blurring capability, Figure 2(a); and then used a multi-level imaging phantom to test the quantitative accuracy, Figure 2(d). The matrix size was $128 \times 128$ for both image phantoms. The photodetector size was $5 \times 5$ pixels. The number of scan lines in the $y$-dimension (horizontal) was 128. The same reconstruction method and parameters were used on both imaging phantoms.
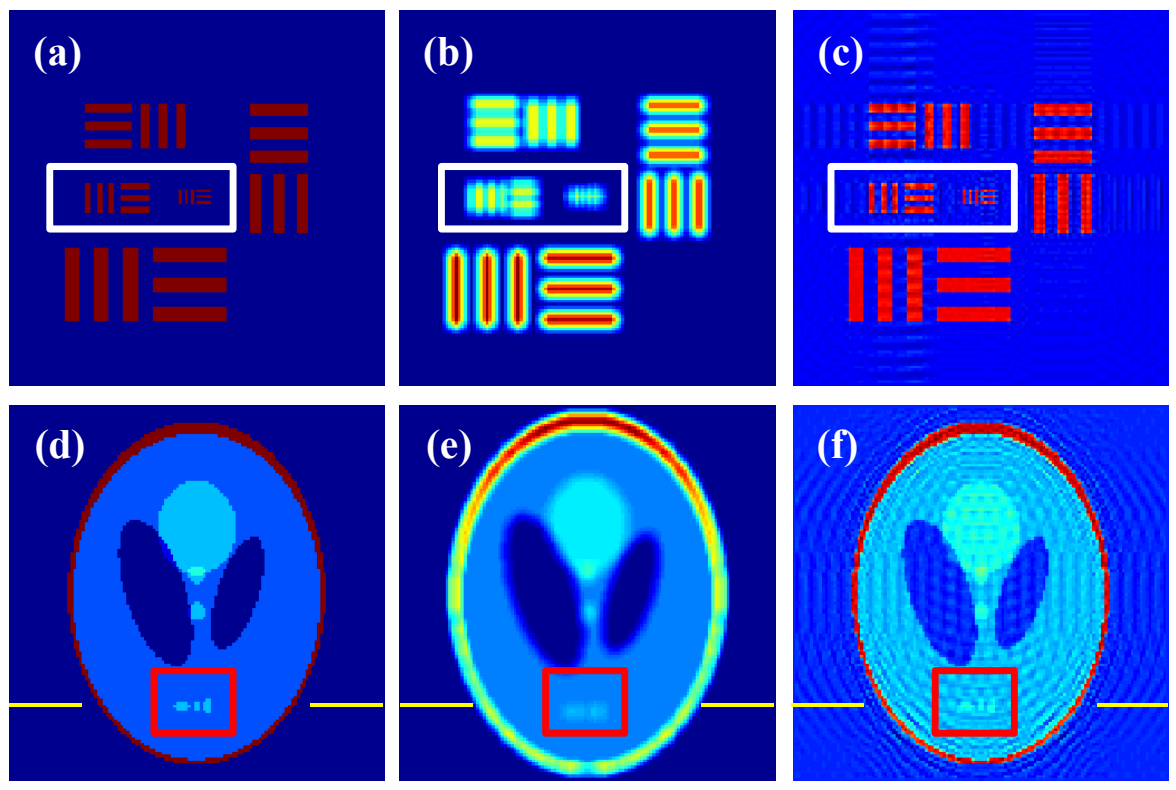

Figure 2. Comparison of the true, blurred, and de-blurred images of two synthesized imaging targets to characterize resolution and quantitation of the proposed super-resolution method. Top raw (a-c): the binary imaging target tests the performance in resolution and qualitative image quality. Bottom raw (d-f): the multi-level imaging target tests the performance in quantitative sampling accuracy. Left column ( $a$ and d): the true images. Middle column (b and e): the blurred images. Right column ( $\mathrm{c}$ and f): reconstruction results using the proposed super-resolution method.

From Figures 2(a-c), one can observe that motion artifact significantly blurred the image, making fine features of the image disappearing; whereas these fine features were effectively recovered using the proposed super-resolution method, shown in the white rectangles in Figures 2(a-c). At the same time, the sharp edges of the stripes in the image were also successfully restored, shown in Figure 2(c). More importantly, although ghosting artifact existed, the intensities of all stripes were practically the same, making the reconstruction a more accurate representation of the true image. 
For the multi-level imaging phantom, comparing Figures 2(d-f), the reconstructed image had similar advantages. More notably, the reconstruction correctly reproduced the outer ring with uniform intensity; although low-level ghosting artifacts exist, the main features of the reconstructed image are free from halo on their edges; the finest features of the phantom were recovered from the blurred image, which were circled by red rectangles in Figures 2(d-f).

A cross-sectional view (along the yellow line near the bottom of these figures) across these fine features revealed the difference of the blurred and recovered features compared to the true image, Figure 3.

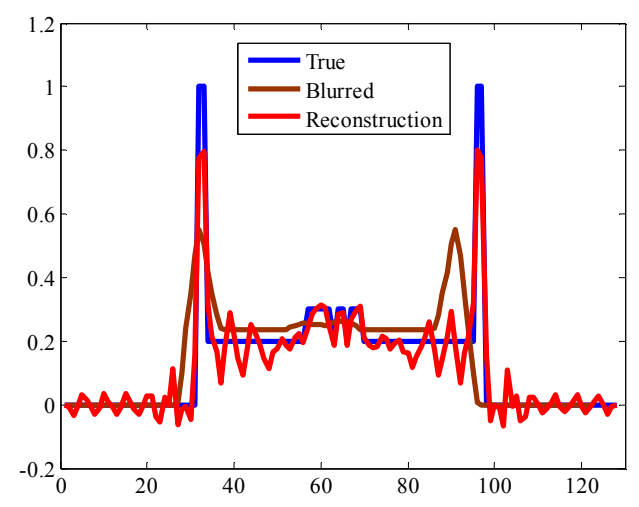

Figure 3. Comparison of the cross-sectional plots across the fine features in the true, blurred, and reconstructed images, drawn along the yellow line near the bottom in Figures 2(d-f). Compared to the blurred image, the reconstruction produced improved results in terms of spatial resolution and quantitative accuracy.

The image qualities of the blurred and reconstructed images were compared to the true image and evaluated with two global metrics: (1) for quantitative comparison of the two sets of blurred and reconstructed images, we computed their standard deviation values (Table 1); and (2) for qualitative evaluation, histograms of the true, blurred, and reconstructed images were drawn, respectively, shown in Figure 4.

Table 1. Quantitative comparison of the blurred and reconstructed images in terms of the standard deviation values from the true image for the binary and multi-level imaging phantoms.

\begin{tabular}{|l|l|l|}
\hline Imaging phantom & Blurred image & Reconstruction \\
\hline Binary phantom & 0.1755 & 0.0520 \\
\hline Multi-level phantom & 0.1263 & 0.0489 \\
\hline
\end{tabular}

Although the two imaging phantoms have different characteristics (binary vs. multi-level and orthogonal vs. circular structures), they produced similar standard deviation values for the blurred and reconstructed images, respectively. This similarly in performance metrics suggested that the proposed super-resolution method were equally effective under these very different conditions. 

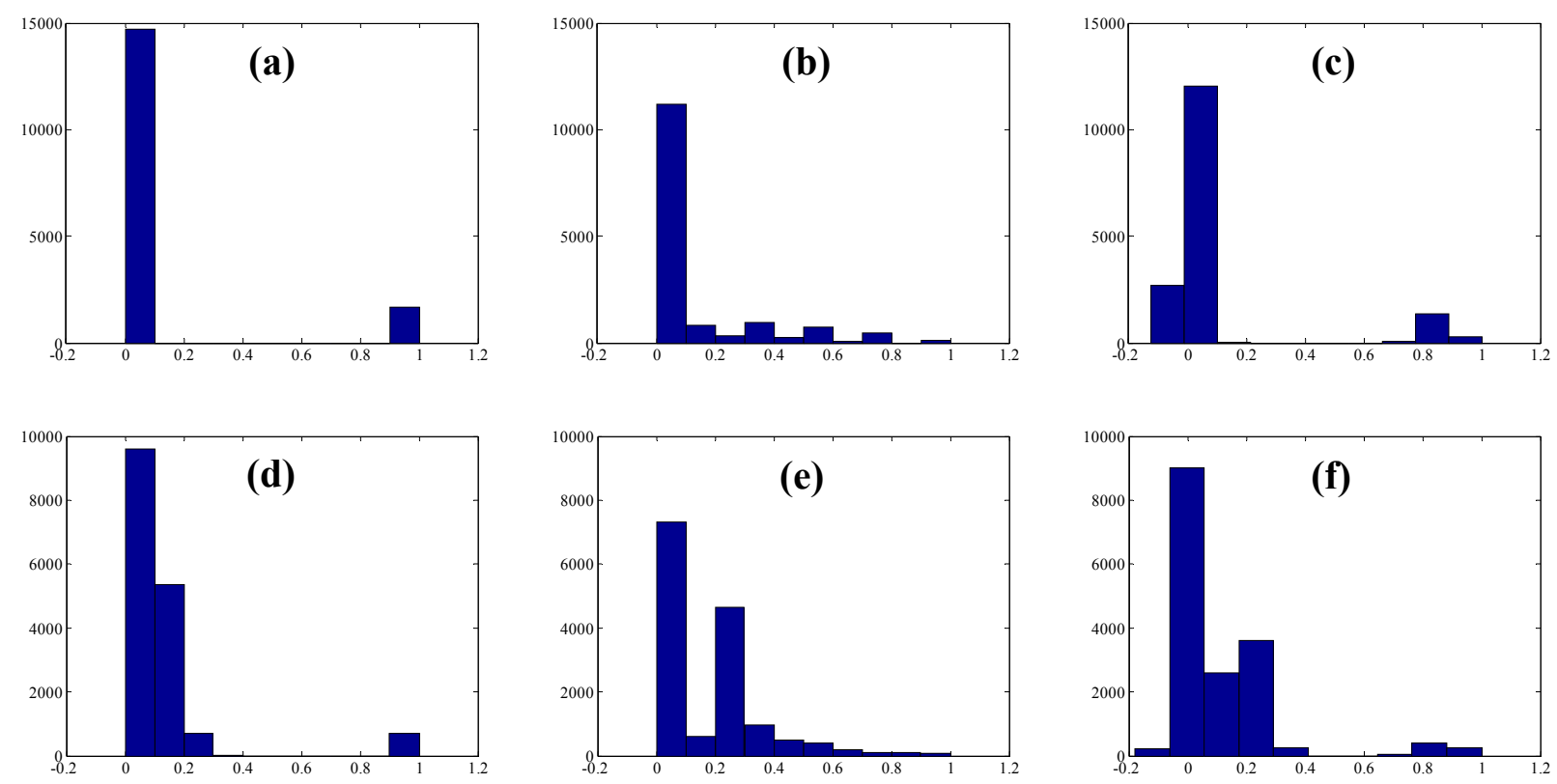

Figure 4. Comparison of the blurred and reconstructed images against the true image using histograms. Top raw (a-c): the binary imaging phantom. Bottom raw (d-f): the multi-level imaging phantom. Left column (a and d): the true images. Middle column ( $b$ and e): the blurred images. Right column ( $c$ and $\mathrm{f}$ ): the reconstructed images.

Summarizing all the data from both two-dimensional imaging phantoms, one can conclude without doubt that the reconstructions using the super-resolution/de-blurring method consistently outperform the uncorrected images. It is noteworthy that reconstruction artifacts resulted in nosier images in both cases compared to their blurred counterparts. However, the improved resolution and quantitative accuracy outweigh this limitation. Furthermore, improved reconstruction algorithms can potentially overcome this limitation. For example, by incorporating a priori information, structural information can be used to assist image reconstruction, e.g., using the methods demonstrated in [10]. In addition, the ghosting artifacts were most prominent around high-intensity sharp edges in the imaging phantoms, e.g., in Figure 2(f), which can potentially be reduced using optimized regularization algorithms [27].

\subsection{Three-dimensional phantom}

In the second set of numerical simulations, a three-dimensional phantom was used to study the capability of the proposed method for diffuse optical imaging. The imaging phantom was a homogenous slab with dimensions of $100 \times 100 \times 15 \mathrm{~mm}$ for width, length, and thickness $(x \times y \times z)$, respectively. The optical properties of the background medium were $\mu_{a}=0.02 \mathrm{~mm}^{-1}, \mu_{s}^{\prime}=1.0 \mathrm{~mm}^{-1}, g=0$ and $n=1.37$. This geometry was designed to simulate the widely used parallel-plate imaging chambers in small animal fluorescence molecular tomography, e.g., [28].

The voxel size of the simulation matrix was $1 \times 1 \times 1 \mathrm{~mm}$. A set of fluorescent inclusions (or, mathematical-equivalently absorbers) were arranged in a square grid pattern of $5 \times 5$ in the equidistant plane between the top and bottom surfaces of the slab. These inclusions had a size of $1 \times 1 \times 1 \mathrm{~mm}$ (i.e., 1 voxel) and separated by $5 \times 5 \mathrm{~mm}$ (center-to-center). A schematic drawing of this three-dimensional imaging phantom is shown in Figure 5.

The excitation light source was positioned at the center of the bottom surface with normal incident angle. The transmitted excitation light was simulated using the Monte Carlo simulation method that we developed previously [29, 30]. The fluorescence signal was computed by multiplying the photon density waves emitted from the source and the detectors, and weighted by the quantum yield of the fluorescent inclusions (assumed unity in this study). 


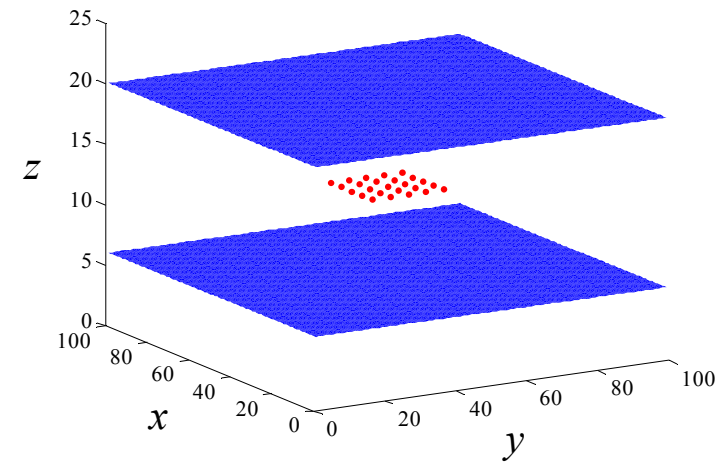

Figure 5. Three-dimensional slab geometry for validating the proposed method in diffuse optical imaging. The slab is homogeneous with significantly larger size in the $x$ - $y$-dimensions than in the $z$-dimension. A grid of $5 \times 5$ inclusions were positioned midway between the top and bottom surfaces to simulate fluorophores.

Bi-directional raster scan was used to measure transmitted fluorescence signal on the top surface. The same characteristics as for the two-dimensional phantoms were assumed for the photodetector. We applied the same superresolution reconstruction algorithm and regularization parameters on the simulated fluorescence data.

Parallel Monte Carlo simulations were performed on clustered computers provided by the Duke Shared Cluster Resource. The computation time is approximately linearly related to the number of available computer cores. In this study, we used 12 cores for 100 million simulated photons, which took typically 10 minutes to complete. Image reconstruction was performed using MATLAB on a computer workstation (Dell Precision 690 with dual Intel Xeon E5320 1.86 GHz 64-bit processors and 32 GB memory). For the image matrix size used in this study (100×100 pixels), reconstruction took approximately $4 \mathrm{~s}$. The proposed reconstruction method successfully produced quantitative correct image from the blurred version, shown in Figure 6.
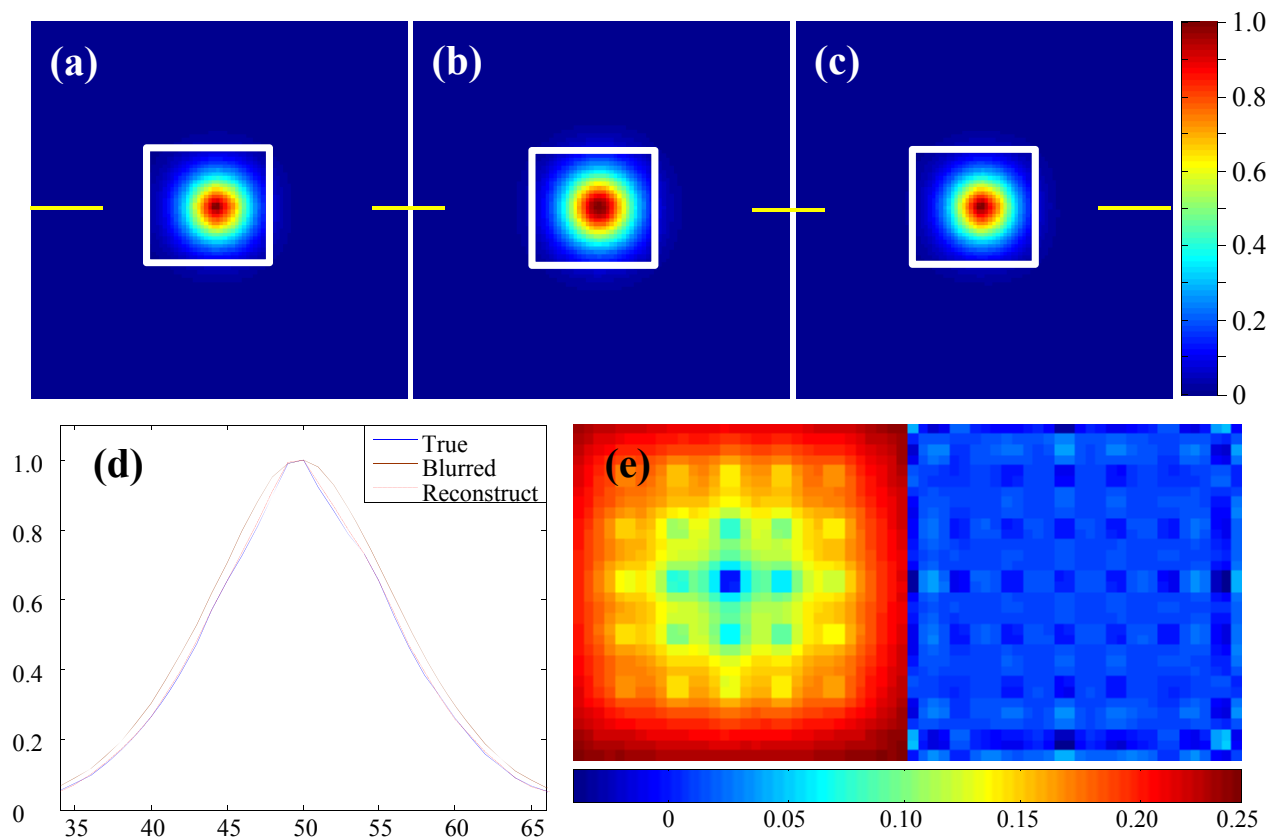

Figure 6. (a) The true, (b) blurred, and (c) reconstructed images of transmittance from the simulated diffuse optical imaging using the geometry shown in Figure 5. (d) Quantitative comparison across the centerline, the yellow broken line within the white rectangles in $(\mathrm{a}-\mathrm{c})$, revealed the differences of the images. (e) Relative error images (defined by the ratio of the image in question to the true image, minus one) of the blurred and reconstructed images, respectively left and right, showed significant improvement of reconstruction (relative error of $17 \pm 4 \%$ vs. $1 \pm 1 \%$, respectively). 
Although the blurred and reconstructed images appear qualitatively similar, Figures 6(a-c), a quantitative comparison across the centerline of these images (along the yellow broken line within the white rectangles) revealed the nuance, Figure 6(d). We defined the relative error image as $A / B-1$, where $A$ is the target image and $B$ is the true image. The error image of the reconstruction, right in Figure 6(e), showed significant improvement compared to the uncorrected data (relative error of $17 \pm 4 \%$ vs. $1 \pm 1 \%$ for the blurred and reconstructed images, respectively, within the same region of interest). In other words, sampling from the super-resolution corrected data have resulted in a significantly reduction in error by more than an order of magnitude compared to the uncorrected data.

For full-field sampling (such is the case of this study), the photodetector scans the entire image plane of the lens. However, in many biomedical applications, only a subset of the image plane needs to be sampled. In diffuse optical imaging, in particular, the effective sampling density is ultimately limited by the thickness (for transmittance measurement) or the depth (reflectance) of the diffusive medium. As a result, the acquisition speed can be expedited by sparse sampling without sacrificing data quality. Our data has shown that without super-resolution reconstruction, the acquired signal is significantly biased regardless of data area-averaging, Figures 6(d and e). This systematic acquisition error will result in severe artifacts in tomographic reconstruction in terms of quantification and resolution.

\section{CONCLUSIONS}

We proposed a new data acquisition method for diffuse optical imaging that uses a scanning photodetector to continuously sample in the image plane of a widefield imaging lens. We developed a super-resolution algorithm to correct the imaging artifacts due continuous acquisition and overlapped spatial transfer function of the photodetector-lens system. Using numerical simulations, we have demonstrated that our acquisition method is effective in optical sampling; and that our super-resolution/de-blurring algorithm significantly improved imaging resolution and quantitative sampling accuracy. With all of the three imaging phantoms that we tested, the super-resolution algorithm consistently produced improved images in terms of resolution and quantitation. With the three-dimensional phantom for diffuse optical imaging, in particular, the reconstructed image offered an acquisition error that was more than an order of magnitude lower than the uncorrected data.

\section{ACKNOWLEDGEMENT}

This project was supported by the National Center for Research Resources and the National Institute of Biomedical Imaging and Bioengineering of the National Institutes of Health through grant numbers R21RR025824 and R21EB015907.

\section{REFERENCES}

[1] Intes, X., et al., "Detection limit enhancement of fluorescent heterogeneities in turbid media by dual-interfering excitation," Appl. Opt. 41(19), 3999-4007 (2002).

[2] Pogue, B.W., et al., "Image analysis methods for diffuse optical tomography," J. Biomed. Opt. 11(3), 33001 (2006).

[3] Kepshire, D.S., et al., "Subsurface diffuse optical tomography can localize absorber and fluorescent objects but recovered image sensitivity is nonlinear with depth," Appl. Opt. 46(10), 1669-1678 (2007).

[4] Zhang, X. and C. Badea, "Effects of sampling strategy on image quality in noncontact panoramic fluorescence diffuse optical tomography for small animal imaging," Opt. Express 17(7), 5125-5138 (2009).

[5] Zhang, X. and A. Webb, "Detectability of absorption and reduced scattering coefficients in frequency-domain measurements using a realistic head phantom," Sensors (Basel) 13(1), 152-164 (2013).

[6] O'Leary, M.A., et al., "Fluorescence lifetime imaging in turbid media," Opt. Lett. 21(2), 158-160 (1996). 
[7] Boas, D.A., A.M. Dale, and M.A. Franceschini, "Diffuse optical imaging of brain activation: approaches to optimizing image sensitivity, resolution, and accuracy," Neuroimage 23(Suppl. 1), S275-S288 (2004).

[8] Gibson, A.P., J.C. Hebden, and S.R. Arridge, "Recent advances in diffuse optical imaging," Phys. Med. Biol. 50(4), R1-R43 (2005).

[9] Cong, W., et al., "Practical reconstruction method for bioluminescence tomography," Opt. Express 13(18), 6756$6771(2005)$.

[10]Zhang, X., C.T. Badea, and G.A. Johnson, "Three-dimensional reconstruction in free-space whole-body fluorescence tomography of mice using optically reconstructed surface and atlas anatomy," J. Biomed. Opt. 14(6), 064010 (2009).

[11] Deliolanis, N., et al., "Free-space fluorescence molecular tomography utilizing 360 degrees geometry projections," Opt. Lett. 32(4), 382-384 (2007).

[12] Da Silva, A., et al., "Optical calibration protocol for an x-ray and optical multimodality tomography system dedicated to small-animal examination," Appl. Opt. 48(10), D151-D162 (2009).

[13] Hyde, D., et al., "Data specific spatially varying regularization for multimodal fluorescence molecular tomography," IEEE Trans. Med. Imaging 29(2), 365-374 (2010).

[14] Venugopal, V., et al., "Full-field time-resolved fluorescence tomography of small animals," Opt. Lett. 35(19), 3189$3191(2010)$.

[15] Kumar, A.T., et al., "A time domain fluorescence tomography system for small animal imaging," IEEE Trans. Med. Imaging 27(8), 1152-1163 (2008).

[16] Nothdurft, R.E., et al., "In vivo fluorescence lifetime tomography," J. Biomed. Opt. 14(2), 024004 (2009).

[17] Leblond, F., et al., "Early-photon fluorescence tomography: spatial resolution improvements and noise stability considerations," J. Opt. Soc. Am. A 26(6), 1444-1457 (2009).

[18] Zhang, X. and C.T. Badea, "Highly efficient detection in fluorescence tomography of quantum dots using time-gated acquisition and ultrafast pulsed laser," Proc. SPIE 7896, 78962W (2011).

[19] Valim, N. and M. Niedre, "Instrumentation considerations for measurement of early arriving photons in diffuse optical tomography," Proc. SPIE 8215, 82150K (2012).

[20] Chaudhari, A.J., et al., "Hyperspectral and multispectral bioluminescence optical tomography for small animal imaging," Phys. Med. Biol. 50(23), 5421-5441 (2005).

[21] Zavattini, G., et al., "A hyperspectral fluorescence system for 3D in vivo optical imaging," Phys. Med. Biol. 51(8), 2029-2043 (2006).

[22] Klose, A.D., "Hyperspectral excitation-resolved fluorescence tomography of quantum dots," Opt. Letts. 34(16), 2477-2479 (2009)

[23] Hernandez-Palacios, J. and L.L. Randeberg, "Intercomparison of EMCCD- and sCMOS-based imaging spectrometers for biomedical applications in low-light conditions," Proc. SPIE 8215, 82150Q (2012).

[24] Heintzmann, R. and M.G.L. Gustafsson, "Subdiffraction resolution in continuous samples," Nat. Photon. 3(7), 362364 (2009).

[25] Greenspan, H., "Super-resolution in medical imaging," The Comp. J. 52(1), 43-63 (2009).

[26]Elad, M. and A. Feuer, "Restoration of a single superresolution image from several blurred, noisy, and undersampled measured images," IEEE Trans. Image Process. 6(12), 1646-1658 (1997).

[27] Jacob, M., et al., "Level-set algorithm for the reconstruction of functional activation in near-infrared spectroscopic imaging," J. Biomed. Opt. 11(6), 064029, (2006).

[28] Graves, E.E., et al., "A submillimeter resolution fluorescence molecular imaging system for small animal imaging," Med. Phys. 30(5), 901-911 (2003).

[29]Zhang, X., V. Toronov, and A. Webb, "Simultaneous integrated diffuse optical tomography and functional magnetic resonance imaging of the human brain," Opt. Express 13(14), 5513-5521 (2005).

[30]Zhang, X., et al., "High-resolution reconstruction of fluorescent inclusions in mouse thorax using anatomically guided sampling and parallel Monte Carlo computing," Biomed. Opt. Express 2(9), 2449-2460 (2011). 